\title{
COSTOS DE PRODUCCIÓN Y COMPETITIVIDAD DEL CULTIVO DE LA PAPA EN EL ESTADO DE MÉXICO ${ }^{1}$
}

\author{
José Luis Morales-Hernández $z^{2}$, Juvencio Hernández-Martínez ${ }^{2}$, Samuel Rebollar-Rebollar², \\ Eugenio Guzmán-Soria
}

\section{RESUMEN}

Costos de producción y competitividad del cultivo de la papa en el Estado de México. El objetivo de esta investigación fue determinar los costos de producción y rentabilidad del cultivo de papa. Esta investigación se realizó de marzo a septiembre de 2009, en el Estado de México y se basó en información obtenida en una encuesta por muestreo estratificado de productores grandes (más de 10 ha), medianos (5-10 ha) y pequeños (1-5 ha) de diez municipios. La metodología utilizada fue la Matriz de Análisis de Política (MAP) la cual tiene como finalidad medir los efectos de la apertura comercial, y la eficiencia de los sistemas de producción. Los resultados mostraron que productores grandes tuvieron mayor rentabilidad $(6.421,0$ a $8.765,0$ US\$) que los productores medianos (5.170,0 a $8.291,0$ US\$) y pequeños (3.107,0 a $6.658,0$ US\$). El cultivo de papa pagó el valor de mercado de los factores internos, incluyendo un retorno al capital. La relación de costo privado en promedio fue menor en productores grandes $(0,24)$ en relación a productores medianos $(0,25)$ y pequeños $(0,30)$ lo cual indicó mayor rentabilidad y competitividad en productores grandes. El consumo intermedio en el ingreso total en productores grandes fue $25 \%$, en tanto, que en medianos y pequeños fue 27,0 y $33 \%$, respectivamente, lo cual integra el porcentaje en la compra de insumo. De manera complementaria el valor agregado en el ingreso total para productores grandes, fue $75 \%$, mientras que para medianos y pequeños fue 73,0 y $67 \%$, representando el aporte del cultivo en la generación de empleo. La actividad productiva del cultivo de papa en 2009, por tanto fue redituable en función de los precios recibidos y pagados.

Palabras clave: Rentabilidad privada, producción papa, ventaja competitiva, costos producción.

\begin{abstract}
Production costs and competitiveness of potato in the state of Mexico. The objective of this work was to determine costs and rentability of potato crop. The study was conducted from March to September 2009 at the state of Mexico, base don the information obtained from surveys with stratified sampling of large producers (more than 10 ha), middle (5-10 ha) and small (1-5 ha) in ten localities. The methodology used was The Matrix of Analysis of Policy (MAP), whose main purpose is to measure the effects of trade liberation and the efficiency of the production systems. The results show that large producers had higher yield (6.421 to 8.765 US\$) than middle-size (5.170 to 8.291 US\$) and small producers (3.107 to 6.658 US\$). Potato farming paid the market price of the internal factors, including a return to the capital. The relation of private costs, on average, was lower in large producers $(0,24)$ than in middle $(0,25)$ and small producers $(0,30)$, which indicates higher yields and competitiveness of large producers. The average consumption in the total income in large producers was $25 \%$, and in middle and small producers was $27 \%$ and $33 \%$ respectively, which integrates the percentage of the purchase of supplies. Complementarily, the added value in the total income, for large producers, was on average $75 \%$, and 73 and $67 \%$ for middle and small producers, which shows the contribution of farming to job creation. The productive activity of potato farming in 2009 was profitable according to the balance of received and paid bills.
\end{abstract}

Keywords: Private profitability, production potato, competitive advantage, costs production

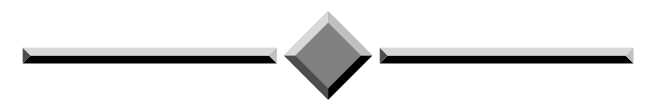

\footnotetext{
1 Recibido: 11 de abril, 2011. Aceptado: 28 de octubre, 2011. Parte de la tesis doctoral del primer autor. Universidad Autónoma del Estado de Mexico. CP 51300.

2 Centro Universitario UAEM Temascaltepec-Universidad Autónoma del Estado de México. Km. 67,5 Carretera Toluca-Temascaltepec. Barrio de Santiago CP 51300.joselui2001@hotmail.com; jh_martinez1214@yahoo.com.mx (autor para correspondencia); samrere@hotmail.com

3 Instituto Tecnológico de Celaya. División de Ciencias Económico Administrativas. Departamento de Ciencias Económicas Administrativas, Posgrado de Administración, Guanajuato, México.eugenio@itc.mx
} 


\section{INTRODUCCION}

A partir de la firma del Tratado de Libre Comercio de América del Norte (TLCAN) en 1994 (Ulloa 2007), en el cultivo de la papa en México, se han presentado contrastes estructurales, desventajas comparativas y competitivas respecto a factores productivos y recursos que manejan los productores de EUA y Canadá; después de quince años de la firma del tratado comercial, estas diferencias no han sido superadas (Gallardo 2007). Consecuencia de ello, el libre comercio en América Latina y en particular en México, ha obligado a los productores de papa, a mejorar su competitividad, ser más eficientes, buscar nuevas fórmulas, vigilar sus costos de producción y adaptarse a las exigencias del mercado. Las diferencias en rendimiento del tubérculo y en inversión, es notoria entre los países firmantes de dicho tratado, por lo que la investigación debería enfocarse hacia la determinación de la competitividad y los costos que la determinan.

Los datos de producción de papa, en el Estado de México, indican que tiene gran importancia socioeconómica, ya que en 2008, contribuyó con 126 mil toneladas, con un valor aproximado de 41 millones de dólares, lo cual lo ubica en cuarto lugar nacional de producción, después de Sinaloa, Sonora y Chihuahua, esta producción la obtuvo en 4554,0 ha, distribuidas en 26 municipios (SEDAGRO 2008).

El potencial de producción en el cultivo de papa en el Estado de México, se estima con base a cambios de tecnología, principalmente en uso de nuevas variedades, manejo de campo y fertilización, lo cual se estima incrementaría los rendimientos hasta 50,0 o $75,0 \%$ (Gallardo 2007) respecto a los actuales niveles productivos de 22,5 t/ha (SAGARPA-SIAP, 2008), mismos que llegarían hasta 32,7 o 38,2 t/ha, y los costos de producción por tonelada se acercarían a los niveles económicos de los E.U. A. y Canadá con valores de 63.5 y 92.0 \$US (Gallardo 2007).

El objetivo principal de este trabajo fue analizar los costos y la competitividad de los productores del cultivo de papa en el Estado de México.

\section{MATERIALES Y METODOS}

La presente investigación se realizó en el Estado de México, en el Centro de la república Mexicana, de latitud norte $22^{\circ} 45^{\prime}$ a $22^{\circ} 55^{\prime}$ y longitud oeste $101^{\circ} 28^{\prime}$ a $105^{\circ} 42^{\prime}$ (Franco et al. 1992). Se realizó en los diez municipios productores de papa más importantes seleccionados en la producción de esta hortaliza (Cuadro 1). Para la operatividad de muestreo, se unieron por pares de acuerdo con su cercanía geográfica donde se consideró necesario.

El análisis de la competitividad se realizó en base a la Matriz de Análisis de Política (MAP) desarrollada por Erick Monke y Scott R. Pearson, de las universidades de Arizona y Stanford (1989), la cual tiene como finalidad medir los efectos de política del gobierno sobre la rentabilidad privada de los sistemas de producción y sobre la eficiencia en el uso de los recursos (Cuadro 2).

Solo se utilizó la primera fila de la matriz (presupuesto privado), por ello sólo se obtuvieron los siguientes indicadores de rentabilidad y competitividad: ganancia o rentabilidad privada (D) $\mathrm{D}=\mathrm{A}-\mathrm{B}-\mathrm{C}$; relación de rentabilidad privada (RRP) $\mathrm{RRP}=\mathrm{D} /(\mathrm{B}+\mathrm{C})$; relación de costo privado ( $\mathrm{RCP}) \mathrm{RCP}=\mathrm{C} /(\mathrm{A}-\mathrm{B})$; consumo intermedio en el ingreso total (PCIP) $\mathrm{PCIP}=\mathrm{B} / \mathrm{A}$; valor agregado en el ingreso total $(\mathrm{PVAP}) \mathrm{PVAP}=(\mathrm{A}-$ B)/A, los cuales se describen a continuación:

\section{Rentabilidad privada o ganancia $(G)$}

La información incorporada en el primer renglón de la MAP permite observar la estimación de la rentabilidad privada. En ésta, la ganancia (D) es la diferencia entre el ingreso privado (A) y el costo de los insumos comerciables, no comerciables y factores internos $(\mathrm{B}+\mathrm{C})$ a precios privados. Esta se estimó con base en precios observados, de mercado o privados; es decir, los ingresos y costos que enfrentaron los productores (incorporan los efectos de las políticas y las distorsiones existentes en los mercados de insumos y productos).

La remuneración del capital, definido como ingreso antes de impuestos que los poseedores requieren para mantener sus inversiones en el sistema, está incluido en los costos de los factores internos (C), por lo que las ganancias (D) están por arriba de los ingresos normales (Monke y Pearson 1989).

De acuerdo con lo anterior, si las ganancias privadas son negativas $(\mathrm{D}<0)$, entonces los productores reciben una tasa de rendimiento menor a la normal, por lo que puede esperarse que abandonen esta actividad, a menos que algún cambio tienda a incrementar las ganancias privadas al nivel normal $(\mathrm{D}=0)$. En contraste, si las ganancias privadas son positivas $(\mathrm{D}>0)$ 
Cuadro 1. Ubicación geográfica y superficie de siembra de papa en Municipios del Estado de México. México. 2009. ${ }^{1}$

\begin{tabular}{lcccc}
\hline Municipio & Latitud norte & Longitud oeste & $\begin{array}{c}\text { Superficie siembra } \\
\text { papa (ha) }\end{array}$ & $\begin{array}{c}\text { Tecnología } \\
\text { SAGARPA** }\end{array}$ \\
\hline Toluca & $19^{\circ} 17^{\prime} 29^{\prime \prime}$ & $99^{\circ} 46^{\prime} 58^{\prime \prime}$ & 20 & RFE, TCF \\
Zinacantepec & $19^{\circ} 17^{\prime} 00^{\prime \prime}$ & $99^{\circ} 44^{\prime} 00^{\prime \prime}$ & 610 & RFE,TMF \\
Tenango & $18^{\circ} 39^{\prime} 7^{\prime \prime}$ & $99^{\circ} 44^{\prime} 00^{\prime \prime}$ & 590 & TMF \\
Calimaya & $18^{\circ} 10^{\prime} 25^{\prime \prime}$ & $99^{\circ} 37^{\prime} 02^{\prime \prime}$ & 105 & TMF \\
Villa Victoria & $19^{\circ} 45^{\prime} 11^{\prime \prime}$ & $99^{\circ} 11^{\prime} 15^{\prime \prime}$ & 350 & TCF \\
Donato Guerra & $19^{\circ} 24^{\prime} 07^{\prime \prime}$ & $100^{\circ} 03^{\prime} 17^{\prime \prime}$ & 10 & TCF \\
San Felipe del Progreso & $19^{\circ} 43^{\prime} 00^{\prime \prime}$ & $99^{\circ} 57^{\prime} 00^{\prime \prime}$ & 200 & TCF \\
Atlacomulco & $19^{\circ} 43^{\prime} 00^{\prime \prime}$ & $99^{\circ} 52^{\prime} 00^{\prime \prime}$ & 80 & TCF \\
San José del Rincón* & - & - & 600 & TMF \\
Juchitepec & $-99^{\circ} 57^{\prime} 00^{\prime \prime}$ & 1125 & TMF \\
Total muestra & & & 3690 & \\
\hline
\end{tabular}

${ }^{1}$ Secretaría de Desarrollo Agropecuario. Estadística 2009, Monografías municipales gobierno del Estado de México. 1990.

* Municipio de creación en 2001, no reportan coordenadas.

** RFE: Riego fertilizado. TMF $=$ Temporal mejorado fertilizado. $\mathrm{TCF}=$ Temporal criollo fertilizado.

Cuadro 2. Estructura de la Matriz de Análisis de Política, según Monke y Pearson 1989. Estado de México, México. 2009.

\begin{tabular}{lcccc}
\hline Concepto & $\begin{array}{c}\text { Ingresos } \\
\text { totales }\end{array}$ & \multicolumn{2}{c}{ Costos de Producción } & Ganancias \\
\cline { 3 - 4 } & & Insumos & $\begin{array}{c}\text { Factores } \\
\text { internos }\end{array}$ & \\
\hline Precios privados & $\mathrm{A}$ & $\mathrm{B}$ & $\mathrm{C}$ & $\mathrm{D}$ \\
Precios económiciables & $\mathrm{E}$ & $\mathrm{F}$ & $\mathrm{G}$ & $\mathrm{H}$ \\
Efectos de política & $\mathrm{I}$ & $\mathrm{J}$ & $\mathrm{K}$ & $\mathrm{L}$ \\
\hline
\end{tabular}

significa que la rentabilidad que están obteniendo es superior a la normal, y ésta debe propiciar una futura expansión del sistema, a menos que otras actividades más rentables en términos privados lo impidan (Monke y Pearson 1989).

\section{Coeficiente de rentabilidad privada (RRP)}

Este indicador se utilizó para conocer el porcentaje de ingreso extraordinario o adicional que recibe el productor por cada dolar invertido. Para su determinación se emplea la relación de ganancia dividida entre la suma de los costos de producción (Monke y Pearson 1989).

\section{Relación del Costo Privado (RCP)}

La competitividad o ganancias privadas se cuantifican a través del indicador conocido como Relación del Costo Privado. Para comparar sistemas que generen productos idénticos, el análisis de las ganancias privadas es insuficiente, pues los resultados de rentabilidad son residuales y podrían provenir de sistemas que utilizan niveles diferentes de insumos para 
producir bienes que también pueden tener diferencias sustanciales en precios. Esta ambigüedad está inherente en las comparaciones de ganancias privadas de sistemas que producen diferentes bienes con variación en intensidad de capital, lo anterior puede evitarse con la estimación de la RCP (Monke y Pearson 1989).

La RCP permite la comparación de eficiencia privada entre diferentes escalas de productores y se obtiene por el cociente del costo de los factores internos (C) y del valor agregado $(\mathrm{A}-\mathrm{B})$.

La RCP indica el límite donde el sistema de producción, en términos de eficiencia, puede sostener el pago de los factores internos (incluyendo el retorno normal del capital) permaneciendo todavía competitivo, esto es, el punto de equilibrio después de obtener ganancias normales, donde $(\mathrm{A}-\mathrm{B}-\mathrm{C})=\mathrm{D}=0$.

Cuando la RCP es menor a la unidad, señala un sistema eficiente y competitivo, toda vez que el valor agregado generado, es suficiente para cubrir el pago de los factores internos, permaneciendo un residuo en dicho valor, que es la retribución a la gestión del productor.

Si la RCP es igual a la unidad, no se generan ganancias extraordinarias, entonces el valor agregado generado por la actividad, solo logra pagar los factores de la producción.

Si la RCP es mayor que la unidad o negativo implica que el sistema productivo es ineficiente y no permite pagar el valor de mercado de los factores internos con el valor agregado generado; además de que la ganancia resulta negativa, por lo que la actividad no es redituable para el productor en función de los precios pagados y recibidos, resultando no competitivo. En general, la minimización de la RCP genera la máxima ganancia privada y se tiene una mayor competitividad (Sosa et al. 2000).

\section{Valor Agregado a Precios Privados (VAP)}

Este indicador se refiere al monto expresado en términos monetarios que permanece en el ingreso recibido, después de haber liquidado el costo de los insumos comerciables y no comerciables, sin tener en cuenta el costo de los factores internos. El valor agregado se valoriza con la retribución de los factores internos, incluida el pago del trabajo del productor. Refleja la contribución de la producción al PIB (Puente 1994).

\section{Consumo Intermedio en el Ingreso Total (PCIP)}

Representa el pago de la actividad hacia el resto de la economía, esto es, son todas aquellas erogaciones que tienen su origen en los ingresos y destinadas para la adquisición de insumos indispensables para impulsar la actividad. Resultan de vital importancia para valorar las actividades agrícolas, ya que demandan insumos y servicios, mano de obra, que son generadas por otros sectores de la economía regional, local o nacional.

\section{Valor Agregado en el Ingreso Total (VPAP)}

Indica el pago o la remuneración de los factores internos de la producción así como la ganancia que obtiene el productor, de tal manera que éste valor refleja el efecto del sistema de producción hacia el interior del propio sector productivo. Con la retribución de los factores internos, se genera empleo e ingreso, los cuales generan a su vez, una demanda por bienes y servicios. (Monke y Pearson 1989).

Para la construcción de la Matriz de Análisis de Política fue necesario elaborar matrices de coeficientes técnicos, de los precios de insumos (comerciables e indirectamente comerciables), de factores internos de la producción y de los precios de producto y subproductos. Los conceptos anteriores se definen brevemente a continuación: Bienes comerciables, son los que tienen un mercado internacional (fertilizantes, semillas, pesticidas, alimentos, vacunas, combustible, detergentes, etc.).

Los insumos indirectamente comerciables, son productos o insumos que se pueden comercializar y, pueden ser también insumos compuestos por partes comerciables y no comerciables dependiendo del bien de que se trate (vehículos, equipamiento, instalaciones), factores internos: son los que intervienen en la producción pero que no son comerciables internacionalmente, tales como mano de obra, energía eléctrica, cuota de agua, administración, seguros y asistencia técnica (Monke y Pearson 1989).

\section{Costos}

En cada estrato de productores se calcularon los valores promedios respecto de los insumos comerciables 
((IC), fertilizantes, fungicidas, insecticidas, semillas, combustible y labores manuales, así como de los factores internos (FI): materiales diversos, renta de la tierra y costos de maquinaria y de administración.

Con los insumos comerciales, se calcula el Costo variable $(\mathrm{CV})$ y con los factores internos e isumos indirectamente comerciables se calculan los Costos Fijos, con los que se obtiene el Costo Total (CT) CT = $\mathrm{CV}+\mathrm{CF}$. Dividiendo CV, CF y CT entre el promedio de rendimiento se obtienen los respectivos costos unitarios por hectárea, $\mathrm{CVu}, \mathrm{CFu}$ y CTu.

El ingreso total unitario se obtiene de dividir el ingreso total por estrato entre el promedio de rendimiento de cada uno. Finalmente la ganancia neta unitaria es la resta de costo total unitario menos ingreso total unitario.

La información de los coeficientes técnicos, así como de las variables productivas del cultivo de la papa en el Estado de México, se obtuvieron a través de 82 encuestas obtenidas mediante un muestreo estratificado dirigido (Cochran 1984). El levantamiento de la información se efectuó de marzo a septiembre de 2009. La estratificación se realizó con base en la superficie que manejan los productores (Santiago y García, 2001): grandes (más de 10 ha), medianos (5-10 ha), y pequeños productores (1-5 ha).

La información obtenida se validó con datos de fuentes secundarias: Fideicomisos Instituidos en relación a la Agricultura (FIRA), Servicio de Información y Estadística Agroalimentaria y Pesquera (SIAP), Secretaría de Agricultura, Ganadería, Desarrollo Rural, Pesca y Alimentación (SAGARPA), Secretaría de Desarrollo Agropecuario del Estado de México (SEDAGRO), Servicio Nacional de Información e Integración de Mercados, Secretaria de Economía (SNIIM), Consejo Nacional Agropecuario (CNA); así como de diversas empresas privadas distribuidoras de insumos agropecuarios, ubicadas en los distintos municipios evaluados.

\section{RESULTADOS Y DISCUSIÓN}

\section{Rendimiento}

Los rendimientos de papa señalan que los productores grandes $(\mathrm{G})$ obtuvieron mayor productividad respecto a los medianos $(\mathrm{M})$ y pequeños $(\mathrm{P})$ (Figura 1); estos datos presentaron significancia estadística $(\mathrm{P}<0,05)$ en rendimiento de tubérculo, de acuerdo con la tecnología utilizada en la producción del mismo. Cabe destacar, que los rendimientos promedio de papa obtenidos por productores grandes, fueron mayores a los que reportó el Servicio de Información Estadística Agroalimentaria y Pesquera (SIAP-SAGARPA 2008), de igual manera, los rendimientos fueron prácticamente similares a los indicados en el paquete tecnológico

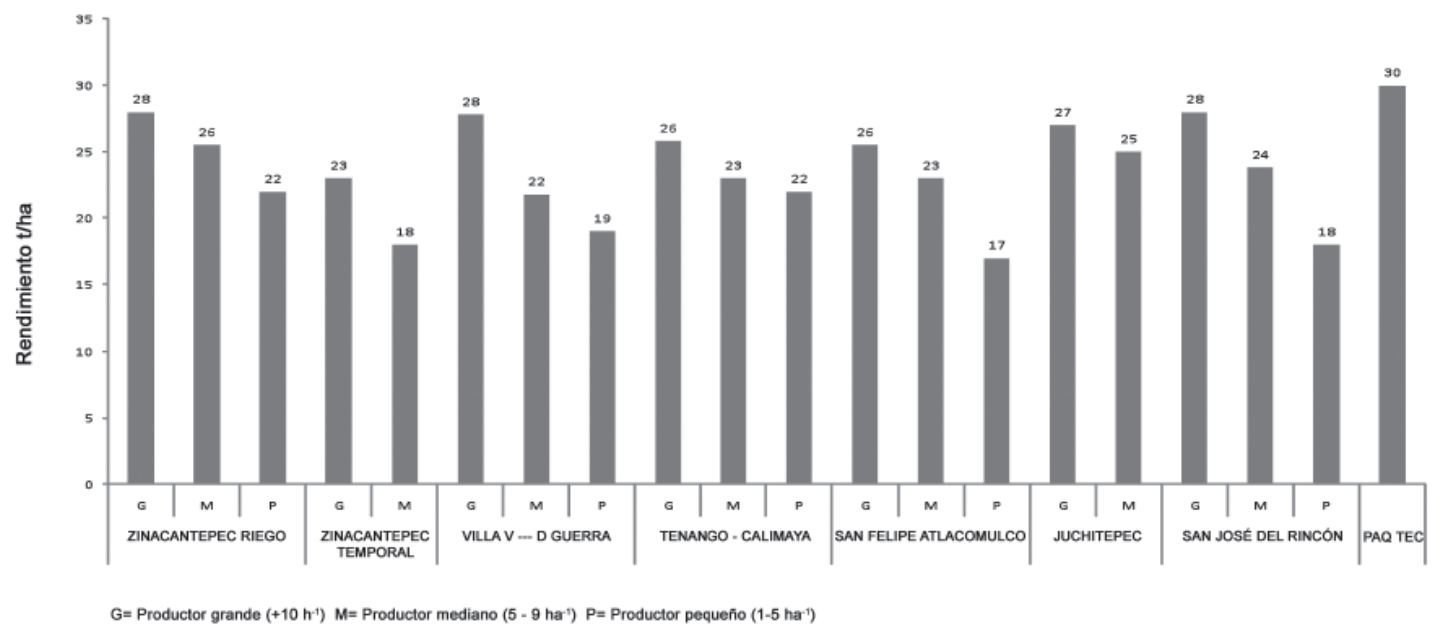

Figura 1. Rendimientos del cultivo de papa por estrato de productores. Estado de México, México. 2009. 
para el Estado de México (Zuloaga 2004) con rangos de 24 a 30 t/ha de producción de tubérculo.

Es importante señalar la similitud de rendimiento de productores grandes en riego en Toluca, y Zinacantepec, respecto al mismo tipo de productores para condiciones de temporal en el resto de municipios, lo cual, es un indicador de que la tecnología en ambas condiciones iguala la productividad del cultivo por ha. Las diferencias de producción entre productores grandes contra medianos y pequeños, revela que el uso de insumos (semilla, pesticidas y fertilización) (Echeverría 2005) y demás factores de la producción fue más eficiente en los primeros.

\section{Costos de producción por hectárea}

El principal componente de los insumos comerciales, fue la semilla, el cual, en productores grandes representó 30,0\% del costo total del cultivo, en tanto que en medianos y pequeños productores fue 28,0 y $33,0 \%$ (Cuadro 3). Los fungicidas e insecticidas representaron $14,0 \%$ del costo del cultivo en productores grandes, $15,0 \%$ en medianos y solo $10,0 \%$ en pequeños productores. La mano de obra, presentó un porcentaje similar en los tres sectores $(14,0-15,0 \%)$, y el uso de materiales diversos (cajas, herramientas, etc.) varió entre 16,0-17,0\% (Cuadro 3).

Cuadro 3. Porcentaje de los insumos comerciables, indirectamente comerciables y factores internos en el cultivo de papa. Estado de México, México. 2009.

\begin{tabular}{lccc}
\hline & $\begin{array}{c}\text { Productores grandes } \\
\text { (más de 10 ha) }\end{array}$ & $\begin{array}{c}\text { Productores medianos } \\
\mathbf{( 5 - 1 0} \text { ha) }\end{array}$ & $\begin{array}{c}\text { Productores pequeños } \\
(\mathbf{1 - 5} \text { ha) }\end{array}$ \\
\hline Fertilizante & $14 \%$ & $15 \%$ & $16 \%$ \\
Fungicida & $3 \%$ & $3 \%$ & $4 \%$ \\
Insecticida & $11 \%$ & $12 \%$ & $6 \%$ \\
Semilla & $30 \%$ & $28 \%$ & $33 \%$ \\
Combustible & $1 \%$ & $1 \%$ & $2 \%$ \\
Labores manuales & $14 \%$ & $14 \%$ & $15 \%$ \\
Labores mecanizadas & $4 \%$ & $3 \%$ & $3 \%$ \\
Materiales diversos & $17 \%$ & $16 \%$ & $16 \%$ \\
Tierra & $4 \%$ & $4 \%$ & $5 \%$ \\
Maquinaria administración y servicios & $3 \%$ & $3 \%$ & $2 \%$ \\
\hline
\end{tabular}

Cuadro 4. Estructura de los costos de producción (en dólares) de papa en el Estado de México, México. 2009.

\begin{tabular}{|c|c|c|c|c|c|c|}
\hline \multirow[t]{2}{*}{ Concepto } & \multicolumn{2}{|c|}{$\begin{array}{c}\text { Productores } \\
\text { grandes (más de } 10 \text { ha) }\end{array}$} & \multicolumn{2}{|c|}{$\begin{array}{c}\text { Productores } \\
\text { medianos (5-10 ha) }\end{array}$} & \multicolumn{2}{|c|}{$\begin{array}{c}\text { Productores } \\
\text { pequeños (1-5 ha) }\end{array}$} \\
\hline & $(\$ / h a)$ & $\%$ & (\$/ha) & $\%$ & (\$/ha) & $\%$ \\
\hline Costos totales & 5641,0 & 100,0 & 5585,0 & 100,0 & 4645,0 & 100,0 \\
\hline Insumos comerciables & 3259,0 & 58,0 & 3334,0 & 60,0 & 2819,0 & 61,0 \\
\hline Factores internos & 2245,0 & 40,0 & 2093,0 & 37,0 & 1709,0 & 37,0 \\
\hline Ins. indirect. comerciables & 137,0 & 2,0 & 148,0 & 3,0 & 116,0 & 3,0 \\
\hline
\end{tabular}


Estos resultados coinciden con los de Hilmig (2003), quien reportó costos de semilla de $30,6 \%$, fungicidas $12,7 \%$, preparación del terreno $12,3 \%$, labores de cosecha $10,4 \%$ y fertilizantes $10,2 \%$. Por otra parte, la industria semillera nacional no abastece más que el $20 \%$ de la demanda actual; por lo cual, el precio de la semilla y el costo de esta por unidad de superficie son elevados para los agricultores (Biarnés et al. 1995). La diferencia del \% insumo semilla en el costo del cultivo en los productores pequeños, se debe a que los grandes, son quienes les venden la semilla con un sobreprecio, en cada municipio.

En términos porcentuales, los costos de producción de papa en el Estado de México, a nivel de estrato, se conformaron mayoritariamente de insumos comerciables $(58,0 ; 61,0$ y $60,0 \%)$ factores internos $(40,0 ; 37,0 ; 37,0 \%)$ e insumos indirectamente comerciables $(3,0 ; 3,0 ; 3,0 \%)$. Los productores medianos y grandes utilizaron cantidades similares de insumos comerciables, mientras que los pequeños utilizan 3,5\% menos en el costo de insumos, con relación al costo productivo de productores grandes (Cuadro 4).

Los costos totales de producción, fueron mayores en productores grandes, le siguieron los medianos y al final los pequeños, con un diferencial de 996,0 dólares entre productores grandes y pequeños (Cuadro 4); estos resultados, fueron similares a los obtenidos en otros Estados de la República Mexicana; por ejemplo, en Coahuila \$US 6.423,0, Michoacán \$5.506,0 y Guanajuato \$4.817,0 (De Santiago, 2007); al mismo tiempo, los valores porcentuales del costo de la semilla en esos estados fueron 26,0, 27,0 y 24,0\%, los cuales resultaron ser menores que el valor porcentual del costo de la semilla en el Estado de México con 28, 30 y $33,0 \%$.

\section{Costos unitarios}

El costo promedio por tonelada de la papa en productores pequeños, se ubicó en \$US 257,0 y fue mayor en $22,0 \%$ con relación al costo que aplicaron los productores grandes, debido a la mayor eficiencia en la aplicación de insumos por parte de estos. A su vez, los costos variables, respecto al costo total, representaron $73,0 \%$ en productores grandes, $76,0 \%$ en medianos, y $75 \%$ en pequeños. El costo fijo por tonelada en productores grandes representó $27,0 \%$ del costo total; tres puntos porcentuales arriba de los medianos y solo un punto respecto a los pequeños productores (Cuadro 5).

Estos resultados pueden ser comparados con los obtenidos en algunos estados de la república mexicana: Coahuila con un $68 \%$ de costo variable y $32 \%$ en costo fijo, Sonora $65 \%$ en costos variables, y $35 \%$ en costos fijos, Sinaloa con $70 \%$ en costos variables y $30 \%$ en costos fijos, Guanajuato con $70 \%$ aplicado en costos variables y $30 \%$ en costos fijos, finalmente Michoacán con $70 \%$ en costos variable, y $30 \%$ en costos fijos (De Santiago 2007).

Los costos unitarios en México son mayores en comparación con los reportados en Estados Unidos 63,5 \$US y Canadá 91,7 \$US/t (Gallardo 2007), es decir, la inversión por tonelada producida en México de 211,0 a 257,0 US\$/t, es el doble o triple que la de los socios del TLCAN. Por tanto, si los costos de producción en México disminuyen, el productor obtendrá una mayor competitividad en el mercado nacional, al mismo tiempo que logre una mayor certeza en la obtención de utilidades (Perea 2008).

Las elevadas diferencias en costos de producción entre Estados Unidos y Canadá, con respecto a México, tienen fundamento en la existencia de subsidios a

Cuadro 5. Costos, ingresos y ganancias (dólares) por tonelada de papa en estratos de productores. Estado de México, México. 2009.

\begin{tabular}{lcccccc}
\hline $\begin{array}{l}\text { Concepto } \\
\text { (promedios) }\end{array}$ & \multicolumn{2}{c}{$\begin{array}{c}\text { Productores grandes } \\
\text { (más de } \mathbf{1 0} \text { ha) }\end{array}$} & $\begin{array}{c}\text { Productores medianos } \\
(\mathbf{5 - 1 0} \mathbf{~ h a )}\end{array}$ & $\begin{array}{c}\text { Productores pequeños } \\
(\mathbf{1 - 5} \text { ha) }\end{array}$ \\
\cline { 2 - 7 } & $\mathbf{( \$ )}$ & $\mathbf{\%}$ & $\mathbf{( \$ )}$ & $\mathbf{\%}$ & $\mathbf{( \$ )}$ & $\mathbf{\%}$ \\
\hline Costo total & 211,0 & 100 & 226,0 & 100 & 257,0 & 100 \\
Costo variable & 154,0 & 73 & 171,0 & 76 & 195,0 & 75 \\
Costo fijo & 57,0 & 27 & 56,0 & 24 & 63,0 & 26 \\
Ingreso total & 497,0 & & 497,0 & & 470,0 & \\
Ganancia neta & 286,0 & & 270,0 & & 213,0 & \\
\hline
\end{tabular}


la agricultura en los dos primeros países, mientras que en este último prácticamente no existen. Los subsidios a la agricultura en Estados Unidos y Canadá represen$\tan 21,0 \%$, mientras en México solo representaron $19,0 \%$ en 2005, para después desaparecer en los años subsecuentes (Gallardo 2007), lo cual fortalece la ventaja competitiva de los EU y de Canadá.

\section{Ingreso total y ganancia neta}

El ingreso por tonelada por estratos de productores analizados, se ubicó en $\$ 497,0, \$ 497,0$ y $\$ 470,0$ (Cuadro 5). Los precios pagados en campo al productor con fluctuación positiva durante 2009 de 0,50 US\$ por kilogramo de tubérculo, influyeron sobre los ingresos mencionados; y de acuerdo a estimaciones de la FAO (2009), los precios pueden mantenerse durante 2010 y 2011, por tanto, este será un indicador que tomará el productor para decidir si cultiva o abandona la producción (Calderón et al. 2004).

Con relación a la ganancia por estrato, los productores grandes y medianos obtuvieron una ganancia similar, en tanto que los pequeños registraron una ganancia menor en 73,0 y 57,0 US\$/t (Cuadro 5), lo cual indica que los productores grandes y medianos presentaron mayor eficiencia en el uso de los insumos; además de otros factores, como el transporte propio, aunado a la comercialización integrada con los compradores del tubérculo.

\section{Rentabilidad privada}

La rentabilidad privada o ganancia refleja las condiciones de tecnología aplicada y muestra las condiciones de mercado en el momento de evaluación, en este sentido, los tres sectores indicaron rentabilidad privada positiva. Por estratos, los grandes obtuvieron la mayor rentabilidad, que se ubicó en $\$ 7.419,0$; le siguieron los medianos con $\$ 6.278,0$ y al final los pequeños productores con rentabilidad de $\$ 4.706,0$ (Cuadro 6).

Tales rentabilidades fueron semejantes a las que reportó De Santiago (2007), en cuatro estados del norte de México, con un rango de 4.053,0 a 8.870,0 US\$.

Estas rentabilidades muestran que este cultivo durante 2009, fue una actividad con ganancia positiva,

Cuadro 6. Rentabilidad a precios privados (dólares) por estrato de productor en el cultivo de papa, en Municipios del Estado de México, México. 2009.

\begin{tabular}{|c|c|c|c|c|c|c|c|c|c|}
\hline \multirow[t]{2}{*}{ Municipio } & \multicolumn{3}{|c|}{$\begin{array}{c}\text { Rentabilidad privada } \\
\text { (\$ dólares) }\end{array}$} & \multicolumn{3}{|c|}{$\begin{array}{c}\text { Relación Rentabilidad } \\
\text { Privada } \%\end{array}$} & \multicolumn{3}{|c|}{$\begin{array}{c}\text { Relación costo } \\
\text { Privado \% } \\
\end{array}$} \\
\hline & $\mathbf{G}^{*}$ & $\mathbf{M}^{*}$ & $\mathbf{P}^{*}$ & G & M & $\mathbf{P}$ & $\mathbf{G}$ & $\mathbf{M}$ & $\mathbf{P}$ \\
\hline \multicolumn{10}{|l|}{ Toluca } \\
\hline Zinacantepec $(\mathrm{R})^{*}$ & 8765,0 & 8291,0 & 6658,0 & 170,0 & 190,0 & 160,0 & 0,17 & 0,18 & 0,20 \\
\hline \multicolumn{10}{|l|}{ Toluca } \\
\hline Zinacantepec $(\mathrm{T})^{*}$ & 6421,0 & 5674,0 & & 128,0 & 110,0 & & 0,25 & 0,29 & \\
\hline \multicolumn{10}{|l|}{ Villa Victoria } \\
\hline D. Guerra & 8189,0 & 6479,0 & 3750,0 & 145,0 & 145,0 & 68,0 & 0,23 & 0,19 & 0,35 \\
\hline Tenango-Calimaya & 7475,0 & 7270,0 & 6485,0 & 139,0 & 175,0 & 146,0 & 0,25 & 0,19 & 0,22 \\
\hline \multicolumn{10}{|l|}{ S. Felipe } \\
\hline Atlacomulco & 6987,0 & 5741,0 & 3107,0 & 121,0 & 97,0 & 58,0 & 0,23 & 0,27 & 0,38 \\
\hline San José del Rincón & 7037,0 & 5320,0 & 3532,0 & 124,0 & 95,0 & 66,0 & 0,27 & 0,28 & 0,36 \\
\hline Juchitepec & 7061,0 & 5170,0 & & 97,0 & 71,0 & & 0,28 & 0,36 & \\
\hline Promedio & 7419,0 & 6278,0 & 4706,0 & 132,0 & 126,0 & 99,0 & 0,24 & 0,25 & 0,30 \\
\hline
\end{tabular}

*T= Temporal, $\mathrm{R}=$ Riego, $\mathrm{G}=$ Productores grandes (más de 10 ha), $\mathrm{M}=$ Productores medianos $(5-10$ ha), $\mathrm{P}=$ Productores pequeños (1-5 ha). 
dada la tecnología utilizada, los precios del producto $\mathrm{y}$ de los insumos que predominaron en el mercado (SNIIM 2009).

\section{Relación de rentabilidad privada}

Dado que esta representa el nivel de ganancia privada (D) como proporción de los costos totales $(\mathrm{B}+\mathrm{C})$, se consideran una remuneración por cada unidad monetaria invertida para financiar los costos totales de operación, en este sentido los productores grandes obtuvieron en promedio $132 \%$, los medianos $126 \%$ y los pequeños obtuvieron un 99\% (Cuadro 6).

\section{Relación de costo privado}

La rentabilidad de la producción de papa entre sectores de productores puede compararse mediante la relación de costo privado ( $\mathrm{RCP})$, con esta se determina la competitividad de las unidades de producción.

La RCP es la rentabilidad evaluada a precios privados y mide la capacidad del sistema para pagar los recursos domésticos, incluyendo un retorno al capital y dejar una utilidad para hacerlo competitivo (Fuentes et al. 1999). En el caso de productores medianos y pequeños el promedio de la RCP fue de 0,25 y 0,30 ; es decir, dado que el grado de competitividad se asoció con el nivel tecnológico y sector de producción
(Jiménez y Quiroz 1999), los productores grandes obtuvieron mejor rentabilidad y competitividad $(0,24)$ lo que significa, que el costo de los recursos internos representó 24,0\% del valor agregado y las ganancias $76,0 \%$ de este (Fuentes et al. 1999) (Cuadro 6). En general, la minimización de la RCP genera la máxima ganancia privada y se tiene una mayor competitividad (Sosa et al. 2000).

\section{Contribución a la economía sectorial y regional}

Los efectos regionales de la producción de papa se observan al desagregar el valor de la producción. Una parte de dicho valor corresponde al consumo intermedio (PCIP), es decir, a las compras y pagos por insumos y servicios de otros sectores productivos de la economía. La segunda parte es el valor agregado (PVAP) del pago a los factores internos de la producción (mano de obra) y la ganancia que obtiene el productor; la cual es un efecto de la producción al interior del sector agrícola.

El consumo intermedio (PCIP) promedio para productores grandes fue del $25 \%$, para medianos y pequeños se ubicó en 27 y 23\%; valores que indican la participación del ingreso en este sector que va hacia el resto de la economía (Cuadro 7).

El valor agregado en el ingreso total (PVAP) promedio para productores grandes fue de $75 \%$, en tanto

Cuadro 7. Valor agregado (dólares) y contribución a la economía del cultivo de papa en Municipios del Estado de México, México. 2009.

\begin{tabular}{lccccccccc}
\hline Municipio & \multicolumn{3}{c}{ Valor agregado (dólares) } & \multicolumn{2}{c}{$\begin{array}{c}\text { Consumo intermedio en } \\
\text { ingreso total (\%) }\end{array}$} & \multicolumn{3}{c}{$\begin{array}{c}\text { Valor agregado insumo total } \\
(\%)\end{array}$} \\
\cline { 2 - 11 } & G* & M* & P* & G & M & P & G & M & P \\
\hline Toluca-Zinacantepec (R)* & 10700,0 & 10029,0 & 8427,0 & 24,0 & 21,0 & 24,0 & 76,0 & 79,0 & 76,0 \\
Toluca .Zinacantepec (T)* & 8609,0 & 8016,0 & & 25,0 & 26,0 & & 75,0 & 74,0 & 73,0 \\
Villa Victoria-D.Guerra. & 10601,0 & 8009,0 & 5796,0 & 23,0 & 27,0 & 38,0 & 77,0 & 62,0 \\
Tenango Calimaya & 9949,0 & 8988,0 & 8321,0 & 23,0 & 21,0 & 24,0 & 77,0 & 79,0 & 76,0 \\
Sn Felipe-Atlacomulco & 8977,0 & 7862,0 & 4983,0 & 29,0 & 32,0 & 41,0 & 77,0 & 68,0 & 59,0 \\
San José del Rincón & 9576,0 & 7411,0 & 5434,0 & 25,0 & 32,0 & 38,0 & 75,0 & 68,0 & 62,0 \\
Juchitepec & 9827,0 & 7849,0 & 0,0 & 29,0 & 35,0 & & 71,0 & 65,0 & 73,0 \\
\hline Promedio & 9748,4 & 8309,1 & 6592,2 & 25,0 & 27,0 & 33,0 & 75,0 & 67,0 \\
\hline
\end{tabular}

*T= Temporal, $\mathrm{R}=$ Riego, $\mathrm{G}=$ Productores grandes (más de 10 ha), $\mathrm{M}=$ Productores medianos $(5-10$ ha), $\mathrm{P}=$ Productores pequeños (1-5 ha). 
en medianos y pequeños productores indicó 73 y $67 \%$ (Cuadro 7).

Estos valores indican la alta participación del cultivo de papa en la generación de empleo, misma que en productores grandes es contratada y en pequeños y medianos es mixta. La suma del consumo intermedio y del valor agregado indicó a su vez, el valor de la producción promedio por hectárea en cada estrato.

En 2009, la producción de la papa en el Estado de México fue una actividad rentable, debido a movimientos favorables en los precios medios rurales del mercado. La rentabilidad privada fue positiva, presentándose el nivel mayor en el estrato de productores grandes, debido al uso oportuno de tecnología y semilla, lo que les permitió obtener altos rendimientos en este tubérculo, situación que favoreció al ingreso y a la ganancia por tonelada producida.

La relación de costo privado (RCP) en los tres estratos de productores de papa en la entidad fue mayor que cero y menor que uno, destacando un menor valor en el estrato de grandes productores, lo que significó una mayor competitividad. Con relación al valor agregado (PVAP), estos se destinaron principalmente a la generación de empleo en la región, mientras que su contribución con el resto de la economía (PCIP) fue menor, ya que solo se destinó a la adquisición de insumos y semillas, participando en menor proporción al ingreso total generado en este cultivo.

\section{LITERATURA CITADA}

Biarnés, A; Colin, JPH; Santiago Cruz, MJ. 1995. Agroeconomía de la papa en México. (en línea). México. Orstom/Colegio de Posgraduados. Consultado 16 dic 2009. Disponible en http://horizon.documentation. ird.fr/exl-docs/pleins_textes/pleins_pleins_textes_7/ b_fdi_03_01/42934.pdf.

Biarnés, A; Colin, JPH; García Mata, R; Santiago Cruz, MJ. 1995. La producción de papa en México. (en línea). Mexico.Orstom/Colegio de Postgraduados. Instituto de Socioeconómica Estadística e Informática. Colegio de Postgraduados Consultado 21 nov 2010. Disponible en http://horizon.documentation.ird.fr/exldocs/pleins_textes/pleins_ pleins_textes_7/b_fdi_03_ 01/42934.pdf.

Calderón Chávez, M; García Mata R; López Díaz, S; Saturnino Mora, FJ; García Salazar, A. 2004. Efecto del precio internacional sobre el mercado de la papa en México 1990-2000. Revista. Fitotecnia Mexicana. Sociedad Mexicana de Fitogenética, A. C. Chapingo, México. 27(4):337-380.

Cochran, W. 1984. Técnicas de muestreo. Ed. C.E.C.S.A. México, D. F. 513 p.

De Santiago, J. 2007. Agroindustria de alto potencial: La protección del mercado encubre el verdadero valor de la papa. Hortalizas México y Centroamérica. (en línea) México. Consultado 10 dic. 2010 Disponible en http://www.hortalizas.com/pdh/?storyd=1143\#.

Echeverría, HE. 2005. Papa. In H.E. Echeverría, F.O. García eds. Fertilidad de Suelos y Fertilización de Cultivos. Ediciones INTA. p. 365-378.

Franco Maass S; Cadena Rivera I; Coronado Ramírez JL; Chacón López O. 1992. Atlas del Estado de México. Gobierno del Estado de México.Universidad Auónoma del Estado de México. Toluca, Méx. 85p.

Fuentes L, FD; Mora, JS; García Mata, R; García, G; Omaña, JM. 1999. Ventaja comparativa y efectos de política en el cultivo de limón en Colima, Oaxaca y Veracruz, México. Agrociencia 33:53-59.

FAO (Organización de las Naciones Unidas para la Agricultura y la Alimentación). Perspectivas Agrícolas OCDE-FAO 2010-19. 2009. (en línea) México. Consulta 18 enero 2010.Disponible en http.//alimentariaonline.com/desplegar_nota.asp?did=6197

Hilmig, V. 2003. Análisis de la producción de papa (Solanum tuberosum L.) en el Estado de Monagas. Revista científica UDO Agrícola 3(1l):74-77.

Jiménez, AL; Quiroz, Y. 1999. Aplicación de la metodología matriz de análisis de política (MAP): El caso de Costa Rica, Secretaría Ejecutiva de Planificación Agropecuaria. XI Congreso Nacional Agronómico 1999. p. 483-493.

Monke, E; Scott, P. 1989. The policy analysis matrix for agricultural development. Cornell University Press. Ithaco. N.Y., USA, and London, England. p. 17-74.

Octavo Congreso Nacional y Cuarto Internacional de la red de Investigación y Docencia sobre Innovación Tecnológica. 2007. Riesgos de la competitividad de los productores mexicanos de papa fresca: Caso Valle del Fuerte, Sinaloa, frente al Tratado de Libre Comercio de América del Norte. Eds. Gallardo Carrillo, PG. Sinaloa, México. p. 1-54

Perea, E. 2009. Suben hasta las papas Sabritas. Imagen Agropecuaria: Agronegocios. (en línea) México. Consultado 31 ene. 2011. Disponible en http://www. imagenagropecuaria.com/secciones.php?id_sec=1 
Puente González, A. 1994. Indicadores económicos de la producción de maíz en México. Instituto Nacional de Investigaciones Forestales y Agropecuarias (INIFAP). Programa de Economía. Publicación especial No 2.32 p.

Santiago Cruz, MJ; García, J. 2001. Economía de la agroindustrialización de la papa en México. Revista Latinoamericana de la papa. Volumen especial. p. 21-43.

SIAP (Servicio de Información y Estadística Agroalimentaria y Pesquera). 2008. Anuario estadístico de la producción agrícola de los Estado Unidos Mexicanos. (en línea). México. Consulta el 7 de septiembre de 2010. Disponible en http://www.siap.gob.mx. Estadísticas por estado. 457 p

SNIIM (Sistema Nacional de Información e Integración de Mercados). 2009. Secretaría de Economía. Precios de mercado de insumos. Consultado enero 2010. Disponible en http://www.economia-sniim.gob.mx/Nuevo/

SEDAGRO (Secretaría de Desarrollo agropecuario del Estado de México) 2008. Oficina estatal de información para el desarrollo rural sustentable. Conjunto Codagem. Estadística de Cultivos: Cultivo de la papa. Conjunto SEDAGRO. Metepec, Estado de México.
SAGARPA-SIAP (Secretaría de Agricultura, Ganadería, Desarrollo Rural, Pesca y Alimentación. Servicio de Información y Estadística Agroalimentaria y Pesquera). 2008. Anuario estadístico de la producción agrícola de los Estados Unidos Mexicanos. Estadísticas por estado. $457 \mathrm{p}$.

Sosa, MM; García, MR; Omaña, SJM; López, DS; López, LE. 2000. Rentabilidad de doce granjas porcícolas en la región noroeste de Guanajuato en 1995. Agrociencia 34:107-113.

Ulloa, B, P. 2007. Promesas y realidades del libre comercio en México: Diez años después del TLC (2004) México sigue viviendo en la pobreza y el subdesarrollo. (en línea). México. Consultado 10 dic. 2009. Disponible en http://sercvicentartículo.blogspot.com/2007/03/ promesas-y-realidades-del-libre.html.

Zuloaga, A. 2004. ICAMEX (Instituto de Investigación y Capacitación Agropecuaria Acuícola y Forestal del Estado de México). Paquetes tecnológicos. 6 ed. Gobierno del Estado de México. Secretaría de Desarrollo Agropecuario. $24 \mathrm{p}$. 